López, A.B., González, I. \& De León, C. (2014). Perfil de un buen docente. Aplicación de un protocolo de evaluación de las competencias del profesorado universitario. Revista Electrónica Interuniversitaria de Formación del Profesorado, 17 (1), 133-148.

DOI: http://dx.doi.org/10.6018/reifop.17.1.190531

\title{
Perfil de un buen docente. Aplicación de un protocolo de evaluación de las competencias del profesorado universitario
}

\author{
Ana Belén López Cámara, Ignacio González López, Carlota de León Huertas \\ Universidad de Córdoba
}

\section{Resumen}

Este trabajo tiene por objetivo establecer un compendio de competencias docentes que las universidades consideran esenciales para definir el rol del profesorado y, a partir de su análisis, establecer un sistema de indicadores de evaluación de la calidad de su actividad docente. A partir de un primer trabajo de diseño de modelo experimental a partir del análisis de los documentos aportados por las unidades de calidad de las universidades españolas y la validación del protocolo por parte de un grupo de expertos de las universidades de Córdoba, Salamanca, Jaume I, Huelva y Sevilla, el modelo resultante se aplicó forma experimental a un grupo de 1316 estudiantes quienes, con sus valoraciones, han permitido elaborar un protocolo de indicadores de evaluación de las competencias docentes del profesorado universitario.

\section{Palabras clave}

Evaluación; competencias docentes; calidad educativa.

\section{Profile of a good teacher. Application of a protocol for the evaluation of the university teacher competencies}




\section{Abstract}

This work aims to establish a compendium of teaching skills that universities consider essential to define the role of teachers and, from their analysis, establish a system of indicators for assessing the quality of their teaching. From an initial design work experimental model based on the analysis of the documents submitted by the departments of Spanish university quality and validation of the protocol by a group of experts from the universities of Cordoba, Salamanca, Jaume I, Huelva and Seville, the resulting model was applied experimentally to a group of 1316 students who, with their values, develop a protocol allowed assessment of indicators of teaching skills of university teachers.

\section{Key words}

Assessment; teaching skills; educational quality.

\section{Introducción}

La adopción de un modelo de formación basado en competencias es uno de los pilares de la nueva expresión educativa universitaria española, derivada de las incitativas tomadas tras la incorporación de nuestro país a la cultura de la convergencia europea. Relacionado con este campo se encuentra ubicado este trabajo, donde el principal objetivo es diseñar un modelo de indicadores de evaluación de la calidad docente del profesorado universitario y crear un protocolo de evaluación de su actividad, a partir de la identificación de las competencias marcadas para tal fin, radicando su utilidad en el autoconocimiento y autoevaluación del profesorado, así como en el diseño y elaboración de propuestas para la mejora de su labor docente.

Según expone Zabalza (2009), la competencia es un constructo molar que sirve para referirnos al conjunto de conocimientos y habilidades que las personas necesitamos para desarrollar algún tipo de actividad. En este sentido, cada actividad suele demandar la presencia de un número determinado y heterogéneo de competencias que pueden posteriormente ser desglosadas en unidades más específicas de competencia en las que se especifican las actividades concretas que están incluidas en la competencia global.

Si nuestro propósito es, entonces, acercarnos a un modelo competencial del rol docente universitario, la tarea se promete compleja, debido a la multiplicidad de aportes y experiencias existentes pero, de entre todas ellas, nos centraremos en las aportadas por Perrenoud (2004), quien propone las siguientes competencias: organización y dinamización de situaciones de aprendizaje, gestión de los progresos en el aprendizaje, empleo de la tecnologías de la información y la comunicación, implicar a los estudiantes en su aprendizaje y esfuerzo, trabajo en equipo y formación de los estudiantes en el trabajo en grupo, afrontar los deberes y dilemas éticos de la profesión, y gestión de su propia formación continua.

Zabalza (2007:106), por su parte, relata de una manera sintética las tres grandes dimensiones en las que se configura el rol docente y que, por tanto, estiman el compendio de competencias constitutivo de la figura del profesor o la profesora:

1. Dimensión profesional, que permite acceder a los componentes clave que definen ese trabajo o profesión: cuáles son sus exigencias (qué se espera que haga ese profesional), cómo y en torno a qué parámetros constituye su identidad profesional, cuáles son los 
principales dilemas que caracterizan el ejercicio profesional en ese ámbito, cuáles son las necesidades de formación inicial y permanente, etc.

2. Dimensión personal, que permite entrar a considerar algunos aspectos de gran importancia en el mundo de la docencia: tipo de implicación y compromiso personal propio de la profesión docente, ciclos de vida de los y las docentes y condicionantes de tipo personal que les afectan (sexo, edad, condición social, etc.), problemas de tipo persona que suelen ir asociados al ejercicio profesional (burn out, estrés, desmotivación, etc.), fuentes de satisfacción e insatisfacción en el trabajo, la carrera profesional.

3. Dimensión laboral que nos sitúa ante los aspectos más claramente relacionados con las condiciones contractuales, los sistemas de selección y promoción, los incentivos, las condiciones laborales (carga de trabajo, horarios, obligaciones vinculadas, etc.).

Acercarnos a la construcción de un sistema competencial completo, complejo e integral supone abordar los procedimientos de la evaluación de la actividad docente para estimar los indicadores relativos a la configuración de un rol docente caracterizado por su calidad. Es sabida la existencia de sistemas de evaluación de la actividad docente en la práctica totalidad de instituciones de educación superior, cuyo objetivo es valorar la calidad de la acción del profesorado e incentivar propuestas de mejora. Se trata de estrategias que pongan en práctica una configuración profesional docente que arbitre una serie de dimensiones que Rué (2004) sentencia como dominio de conocimientos propios de su ámbito formativo, desarrollo del conocimiento científico, habilidades de resolución de problemas, capacidad de trabajo en grupo, orientación ética del trabajo profesional y formación continua.

La implementación de estas propuestas evaluativas no debe suponer la aplicación de una serie de pruebas llevadas a cabo puntualmente, sino un proceso continuo y sistemático encaminado, desde el punto de vista de Jornet et al. (1988), a ayudar a cada uno de los docentes en su desarrollo profesional y en la planificación de su carrera, así como contribuir a que la formación en ejercicio se ajuste a las necesidades reales del profesorado.

A pesar de que la evaluación es considerada una tarea compleja y, en ocasiones, conflictiva, la evaluación de la actividad docente del profesorado universitario sigue siendo constante objeto de estudio. Tal es así, que ha pasado de valorar únicamente la adquisición o no de conocimientos por parte de los estudiantes, a considerar este sistema como un elemento esencial en el análisis de la calidad de las instituciones educativas. Es una tarea tan importante, que uno de los elementos condicionantes de la calidad de los centros docentes universitarios, tal y como ya señaló en el año 1995 en el Plan Nacional de Evaluación de la Calidad de las Universidades, es la docencia como factor clave del conjunto de la calidad del proceso de enseñanza/aprendizaje. Esta docencia, es evaluada por las diferentes propuestas que siguen las Administraciones Públicas de nuestro país (VERIFICA, AUDIT, ACREDITA y DOCENTIA), donde podemos destacar cuatro modelos de evaluación dentro de los que destaca la actividad docente del profesorado como una de sus dimensiones: EFQM, PEI, AUDIT y DOCENTIA.

Ante ello, Tejedor y Jornet (2008) consideran que el modelo global de evaluación del subsistema profesorado tendrá que estar relacionado, cuando menos, con los siguientes aspectos y fuentes de información:

- Evaluación de la actividad instructiva (aula y tutorías). Autoinforme del profesor, opinión de las autoridades académicas del centro y opinión de los alumnos. 
- Evaluación de la actividad investigadora, a partir de indicadores relacionados con la calidad, la cantidad y la utilidad social y académica (incorporación de los resultados de investigación y de las aportaciones metodológicas a la actividad instructiva).

- Evaluación de la actividad departamental.

- Evaluación, con carácter complementario, de la actividad relacionada con la prestación de servicios a la comunidad (universitaria, profesional y social).

- Evaluación de las condiciones de trabajo del profesor. Para obtener una valoración realista, sin sesgo, deberán considerarse las condiciones académicas, personales e institucionales en las que se desarrolla el trabajo del profesor evaluado. Esto incluye su status profesional, organización académica, disponibilidad de recursos para docencia e investigación, programas de formación docente de la institución y características de los alumnos, con el fin de contextualizar ampliamente el proceso de evaluación del profesorado en sentido amplio.

Con todo ello, es preciso revisar, tal y como lo argumenta Fueyo (2004) las tendencias que se están asentando en el ámbito universitario con el fin de frenar las perversas consecuencias que van a tener a la larga en la vida universitaria en general y plantear alternativas más próximas a la evaluación de la actividad de los y las docentes en su función de intelectuales e investigadores ligados a contextos determinados con los que asumen un compromiso y no una mera relación burocrática.

\section{Metodología}

Con esta investigación pretendemos, en un primer momento, advertir cuáles son las competencias docentes que las universidades españolas consideran esenciales para definir el rol del profesorado universitario y, a partir de su análisis, establecer un sistema de indicadores de evaluación de la calidad docente, ya que son aceptados por su carácter sintético y su capacidad para orientar la toma de decisiones (Tiana Ferrer, 1998).

Las 53 unidades técnicas de calidad de las diferentes instituciones universitarias de carácter público con las que contactamos, obteniendo respuesta de sólo 32 de ellas, a quienes les fueron solicitados los instrumentos actualmente en uso para llevar a cabo la evaluación docente. Es de suponer que dichos sistemas cuentan con las garantías científicas pertinentes de fiabilidad y validez, así como de adaptación a las características contextuales, sociales y educativas de las diferentes universidades.

A partir de las dimensiones analizadas, han sido identificadas las competencias a valorar y se determinaron los indicadores pertinentes para cada una de ellas, en función de los cuales procedimos a la construcción de un protocolo de evaluación de la labor docente del profesorado universitario.

Han sido básicamente dos los momentos de relevancia que han dado como resultado la elaboración del protocolo de evaluación citado; el primero de ellos consiste en el análisis exhaustivo de la información recogida de los instrumentos que nos aportaron las diferentes universidades españolas, comenzando por su denominación, así como por un estudio de los sistemas de medición empleados, las dimensiones consideradas y los indicadores desarrollados. En un segundo momento y tras la construcción de un borrador, este fue sometido a valoración por parte de una serie de expertos y expertas de cinco universidades (Córdoba, Salamanca, Huelva, Sevilla y Jaume I) que procedieron a valorar la pertinencia y 
claridad de los elementos propuestos, así como la definición e idoneidad de las dimensiones en los que estos han sido agrupados.

Tabla 1: Indicadores de evaluación competencial del profesorado universitario

\begin{tabular}{|c|c|}
\hline DIMENSIONES & INDICADORES \\
\hline \multirow{7}{*}{$\begin{array}{l}\text { Diseño de los } \\
\text { programas/guías } \\
\text { docentes de la } \\
\text { asignatura }\end{array}$} & 1. Adecuación de los objetivos con el perfil profesional de la titulación \\
\hline & $\begin{array}{l}\text { 2. Vinculación de los contenidos con los diferentes aspectos de la } \\
\text { profesión }\end{array}$ \\
\hline & 3. Adecuación de las competencias con el desarrollo de la profesión \\
\hline & $\begin{array}{l}\text { 4. Adecuación de la metodología de enseñanza con las diferentes tareas } \\
\text { de aprendizaje }\end{array}$ \\
\hline & $\begin{array}{l}\text { 5. Adecuación de los sistemas de evaluación empleados con las diferentes } \\
\text { tareas propuestas }\end{array}$ \\
\hline & $\begin{array}{l}\text { 6. Adecuación de la bibliografía y los recursos con las finalidades de la } \\
\text { asignatura }\end{array}$ \\
\hline & $\begin{array}{l}\text { 7. Existencia de sistemas de coordinación de las actividades teóricas y } \\
\text { prácticas previstas en el programa }\end{array}$ \\
\hline \multirow[t]{15}{*}{$\begin{array}{l}\text { Metodología } \\
\text { docente }\end{array}$} & $\begin{array}{l}\text { 8. Organización y presentación de los materiales adecuada (explicaciones, } \\
\text { materiales escritos, actividades, etc.) }\end{array}$ \\
\hline & $\begin{array}{l}\text { 9. Coherencia entre los objetivos y contenidos de la asignatura y las } \\
\text { actividades realizadas }\end{array}$ \\
\hline & 10. Aporte de bibliografía y/o fuentes de información relevantes \\
\hline & 11. Relación entre los distintos bloques temáticos de la materia \\
\hline & $\begin{array}{l}\text { 12. Coordinación entre el profesorado para evitar solapamientos y } \\
\text { relacionar los contenidos de las distintas asignaturas }\end{array}$ \\
\hline & 13. Seguimiento del proceso de aprendizaje del alumnado \\
\hline & 14. Fomento de la participación en clase y motivación al alumnado \\
\hline & 15. Desarrollo de la capacidad de síntesis y de razonamiento del alumnado \\
\hline & $\begin{array}{l}\text { 16. Uso de metodologías para implicar activamente a los estudiantes en el } \\
\text { aprendizaje }\end{array}$ \\
\hline & $\begin{array}{l}\text { 17. Organización en la exposición, destacando los contenidos más } \\
\text { importantes }\end{array}$ \\
\hline & $\begin{array}{l}\text { 18. Exposición de ejemplos en los que se ponen en práctica las } \\
\text { competencias a desarrollar por la asignatura }\end{array}$ \\
\hline & 19. Promoción del desarrollo en el alumno de una actitud reflexiva \\
\hline & $\begin{array}{l}\text { 20. Propuesta de actividades para favorecer el aprendizaje autónomo } \\
\text { (búsqueda de información complementaria, trabajos, investigaciones, } \\
\text { etc.) }\end{array}$ \\
\hline & 21. Uso de un lenguaje claro, preciso y riguroso \\
\hline & $\begin{array}{l}\text { 22. Reparar en la opinión de los estudiantes en cuestiones relacionadas con } \\
\text { el desarrollo de la docencia }\end{array}$ \\
\hline \multirow{3}{*}{$\begin{array}{l}\text { Actividades } \\
\text { prácticas }\end{array}$} & 23. Adecuación de las prácticas al contexto teórico de la asignatura \\
\hline & 24. Adecuación del número de prácticas al desarrollo de la asignatura \\
\hline & $\begin{array}{l}\text { 25. Utilidad de las prácticas para el desarrollo de competencias } \\
\text { profesionales }\end{array}$ \\
\hline \multirow[t]{3}{*}{$\begin{array}{l}\text { Recursos } \\
\text { didácticos }\end{array}$} & $\begin{array}{l}\text { 26. Uso de recursos adecuados (pizarra, transparencias, medios } \\
\text { audiovisuales, material de apoyo en red virtual...) que facilitan el } \\
\text { aprendizaje }\end{array}$ \\
\hline & $\begin{array}{l}\text { 27. Elaboración de manuales didácticos de apoyo al desarrollo de la } \\
\text { asignatura }\end{array}$ \\
\hline & 28. Diseño de cuadernos de prácticas para su implementación en el aula \\
\hline \multirow{2}{*}{$\begin{array}{l}\text { Sistemas de } \\
\text { evaluación }\end{array}$} & 29. Información del sistema de evaluación al comienzo del curso \\
\hline & 30. Información del sistema de evaluación durante el transcurso de la \\
\hline
\end{tabular}




\begin{tabular}{|c|c|}
\hline \multirow[t]{4}{*}{ 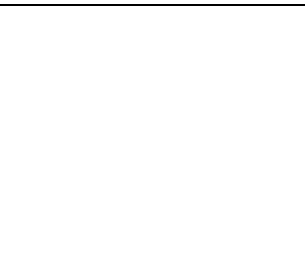 } & docencia \\
\hline & 31. Información del sistema de evaluación al finalizar el curso \\
\hline & $\begin{array}{l}\text { 32. Evaluación coherente con los objetivos, contenidos, metodología y } \\
\text { actividades del curso }\end{array}$ \\
\hline & $\begin{array}{l}\text { 33. Variedad de procedimientos para evaluar el aprendizaje de los } \\
\text { alumnos/as }\end{array}$ \\
\hline \multirow{7}{*}{$\begin{array}{l}\text { Actitud } \\
\text { profesorado }\end{array}$} & 34. Actitud receptiva en su relación con el alumnado \\
\hline & 35. Respeto hacia el alumnado \\
\hline & 36. Interés por la asignatura por parte del docente \\
\hline & 37. Atención ante las dificultades de aprendizaje de sus estudiantes \\
\hline & $\begin{array}{l}\text { 38. Estímulo al alumnado para que se interese por su proceso de } \\
\text { aprendizaje }\end{array}$ \\
\hline & 39. Creación de un clima de trabajo y participación \\
\hline & 40. Promoción de una comunicación fluida y espontánea \\
\hline
\end{tabular}

Los indicadores establecidos por parte del grupo de expertos y expertas en la fase anterior (ver tabla 1) han sido convertidos en ítems de valoración escalar, dando lugar, al segundo momento que ha sido la elaboración de una encuesta que posteriormente se ha implementado a un total de 1316 estudiantes a quienes se ha solicitado su valoración para la construcción de un modelo exhaustivo de evaluación de las competencias docentes del profesorado universitario, emanadas de la incorporación de las universidades españolas en el Espacio Europeo de Educación Superior.

La muestra de estudiantes que han participado en este estudio ha sido eminentemente femenina (63,8\%), siendo la edad media de este alumnado de 21,75 años, correspondiendo la edad mínima a 18 y la máxima a 69. La distribución del alumnado en función de la Universidad y de la macroárea en la que se encuentra matriculado es la que se aprecia en la tabla 2, donde podemos observar que hemos contado con la participación de estudiantes de las cinco universidades que son corresponsables de este proyecto. Sin embargo, y dada la especificidad de cada una de ellas, su representatividad en este estudio nos es totalmente homogénea aunque sí significativa para los procedimientos analíticos que darán lugar al protocolo de evaluación final. La información que presenta la tabla 1 muestra que un 35,6\% del alumnado participante procede de la Universidad de Córdoba, seguido en igualdad de condiciones por las instituciones de Sevilla (19,7\%) y Huelva (11,9\%). En último lugar están representadas la Universidades de Salamanca (13,5\%) y Jaume I de Castellón $(11,9 \%)$.

Asimismo y tratando de revelar el carácter académico de cada institución participante, hemos procedido a realizar una tabla de contingencia que nos informe sobre la presencia de cada macroárea en las universidades participantes, pudiéndose apreciar que la Universidad de Córdoba aporta al estudio una representación equitativa en cada una de las macroáreas en las que se clasifican las titulaciones universitarias. Lo mismo ocurre en la Universidad de Huelva, aunque hay que señalar que ha sido escasa la participación del alumnado procedente de las titulaciones experimentales. Encontramos, asimismo, que la Universidad de Sevilla participa con estudiantes en todas las ramas de especialización a excepción de titulaciones de Humanidades. Por otro lado, desde la Universidad Jaume I de Castellón observamos que el 50\% del alumnado participante pertenece a titulaciones Jurídico-Sociales, no existiendo representación de carreras de Ciencias de la Salud y Experimentales. Por último, hay que destacar que la Universidad de Salamanca participa con una representación similar de alumnos y alumnas de Humanidades y Ciencias JurídicoSociales. 
Tabla 2: Distribución del alumnado en función de la Universidad y la macroárea

\begin{tabular}{|c|c|c|c|c|c|c|c|c|c|c|c|c|}
\hline \multirow{3}{*}{ Universidad } & \multicolumn{10}{|c|}{ MACROÁREA } & \multirow{2}{*}{\multicolumn{2}{|c|}{ Total }} \\
\hline & \multicolumn{2}{|c|}{$\begin{array}{l}\text { Humani- } \\
\text { dades }\end{array}$} & \multicolumn{2}{|c|}{$\begin{array}{l}\text { Jurídico- } \\
\text { Sociales }\end{array}$} & \multicolumn{2}{|c|}{$\begin{array}{c}\text { Ciencias de } \\
\text { la Salud }\end{array}$} & \multicolumn{2}{|c|}{$\begin{array}{l}\text { Experime } \\
\text { ntales }\end{array}$} & \multicolumn{2}{|c|}{ Técnicas } & & \\
\hline & $\mathbf{F}$ & $(\%)$ & $\mathbf{F}$ & $(\%)$ & $\mathbf{F}$ & $(\%)$ & $\mathbf{F}$ & $(\%)$ & $\mathbf{F}$ & (\%) & $\mathbf{F}$ & (\%) \\
\hline Córdoba & 86 & 18,3 & 97 & 20,7 & 117 & 24,9 & 68 & 14,5 & 101 & 21,5 & 469 & 100 \\
\hline Huelva & 53 & 20,9 & 84 & 33,1 & 67 & 26,4 & 8 & 3,1 & 42 & 16,5 & 254 & 100 \\
\hline Sevilla & 0 & 0,0 & 80 & 30,9 & 97 & 37,5 & 52 & 20,1 & 30 & 11,5 & 259 & 100 \\
\hline Jaume i & 28 & 17,9 & 78 & 50,0 & 0 & 0,0 & 2 & 1,3 & 48 & 30,8 & 156 & 100 \\
\hline Salamanca & 97 & 54,5 & 81 & 45,5 & 0 & 0,0 & 0 & 0,0 & 0 & 0,0 & 178 & 100 \\
\hline
\end{tabular}

Tratando de especificar el carácter de género de cada una de las macroáreas y haciendo, por ello, una tabla de contingencia, comprobamos que los hombres cursan en primer lugar carreras de tipo técnico, seguidos de especialidades jurídico-sociales. Sin embargo, las mujeres se decantan en primera opción con carreras de la macroárea jurídico-social, eligiendo en segunda opción titulaciones relacionadas con las ciencias de la salud.

En último lugar, queremos hacer constar que existe un elevado porcentaje de alumnos y alumnas matriculado en los tres primeros cursos, debido a que todas las titulaciones universitarias comportan estos niveles. Asimismo, se aprecia una baja relevante en la representación de los últimos tres niveles, debido a que se encuentran únicamente en las licenciaturas.

\section{Resultados}

Presentamos, a continuación, las valoraciones realizadas por el alumnado a los diferentes indicadores propuestos, atendiendo a las dimensiones en lo que estos quedan clasificados. Queremos hacer constar que, tras aplicar una prueba de $t$ de Student (n.s.=0.05) para comprobar la existencia de diferencias estadísticamente significativas atendiendo al sexo del alumnado, los resultados obtenidos concluyen que esta variable no incide en la existencia de matices que provoquen alguna diferenciación en ninguna dimensión, por lo que la opinión de ambos grupos es estadísticamente similar en todos los aspectos valorados.

\section{Guía docente}

La Guía Docente se constituye en una herramienta básica del Sistema Europeo de Transferencia de Créditos (ECTS) para alcanzar el objetivo de "promover la cooperación europea en garantía de calidad mediante el desarrollo de metodologías y criterios comparables" (Declaración de Bolonia, 1999). La Guía Docente es lo que Salinas y Cotino (2005) denominan una planificación detallada de cualquier asignatura o módulo basada en los principios que orientan el proceso de Convergencia en la creación de un Espacio Europeo de Educación Superior. Si en otro tipo de programas de asignaturas el eje se situaba sobre el contenido (selección de contenidos, su estructura y distribución en el programa, criterios para su evaluación, etc.), en este caso el eje es doble: el contenido y el trabajo del estudiante en torno a ese contenido. Desde esta perspectiva se entiende que la docencia no solo se puede limitar a la actividad en el aula.

Una aproximación a la valoración que realiza este alumnado a los elementos que conforman esta dimensión aparece reflejada en la tabla 3. En ella se observa cómo este grupo de estudiantes considera relevantes para el diseño de una guía docente una bibliografía y unos recursos adecuados a las finalidades de la materia, una vinculación directa con el perfil profesional al que se dirigen sus estudios, lo que lleva a una buena 
concreción y coordinación de actividades teóricas y prácticas, una metodología adecuada y unos correctos sistemas de evaluación.

Tabla 3: Valoración de la dimensión "Diseño de los programas / guías docentes"

\begin{tabular}{|l|c|c|c|}
\hline \multicolumn{1}{|c|}{ DISEÑO DE LOS PROGRAMAS / GUíAS DOCENTES } & Media & $\mathbf{S}_{\mathbf{x}}$ & $\mathbf{N}$ \\
\hline Adecuación de los objetivos con el perfil profesional de la titulación & 3,42 &, 951 & 1307 \\
\hline $\begin{array}{l}\text { Vinculación de los contenidos con los diferentes aspectos de la } \\
\text { profesión }\end{array}$ & 3,49 &, 925 & 1304 \\
\hline Adecuación de las competencias con el desarrollo de la profesión & 3,40 &, 951 & 1301 \\
\hline $\begin{array}{l}\text { Adecuación de la metodología de enseñanza con las diferentes } \\
\text { tareas de aprendizaje }\end{array}$ & 3,36 &, 952 & 1296 \\
\hline $\begin{array}{l}\text { Adecuación de los sistemas de evaluación empleados con las } \\
\text { diferentes tareas propuestas }\end{array}$ & 3,35 &, 986 & 1299 \\
\hline $\begin{array}{l}\text { Adecuación de la bibliografía y los recursos con las finalidades de la } \\
\text { asignatura }\end{array}$ & 3,52 & 1,014 & 1307 \\
\hline $\begin{array}{l}\text { Existencia de sistemas de coordinación de las actividades teóricas y } \\
\text { prácticas previstas en el programa }\end{array}$ & 3,41 & 1,059 & 1305 \\
\hline
\end{tabular}

Una vez realizada esta primera aproximación, hemos querido establecer algún matiz diferencial en función de la Macroárea y de la universidad en la que se encuentra matriculado el alumnado, para lo que hemos aplicado un Análisis de Varianza de un Factor (n.s.=0.05) y un contraste Post-Hoc HSD de Tukey que nos explicita cuáles son los grupos entre los que se evidencian esas diferencias. Los datos de la tabla 4 reflejan lo siguiente:

- El indicador referente a la "adecuación de los objetivos con el perfil profesional de la titulación" es valorado positivamente por todo el alumnado, sin diferencias entre las universidades.

- El alumnado de la Universidad de Sevilla valora muy positivamente la "vinculación de los contenidos con los diferentes aspectos de la profesión", la "adecuación de las competencias con el desarrollo de la profesión" y la "adecuación de la bibliografía y los recursos con las finalidades de la asignatura".

- El alumnado de la Universidad de Huelva considera, entre otros aspectos, la relevancia de la "vinculación de los contenidos con los diferentes aspectos de la profesión, la "adecuación de la metodología de enseñanza con las diferentes tareas de aprendizaje", la "adecuación de los sistemas de evaluación empleados con las diferentes tareas propuestas", la "adecuación de la bibliografía y los recursos con las finalidades de la asignatura" y la "existencia de sistemas de coordinación de las actividades teóricas y prácticas previstas en el programa”

- El alumnado de la Universidad Jaume I considera de relevancia los aspectos relacionados con la "vinculación de los contenidos con los diferentes aspectos de la profesión", la "adecuación de las competencias con el desarrollo de la profesión", la "adecuación de la metodología de enseñanza con las diferentes tareas de aprendizaje" y la "adecuación de los sistemas de evaluación empleados con las diferentes tareas propuestas".

- El alumnado de la Universidad de Salamanca considera igualmente relevante el indicador que hace referencia a la "adecuación de la bibliografía y los recursos con las finalidades de la asignatura" 
- El alumnado de Ciencias de la Salud considera relevante, a diferencia del resto de especialidades, "Vincular los contenidos con los diferentes aspectos de la profesión".

- El alumnado de titulaciones de Humanidades y Ciencias Experimentales valoran muy positivamente "adecuar la bibliografía y los recursos con las finalidades de la asignatura".

\section{Metodología docente}

La metodología docente responde a cómo un docente puede proceder con su alumnado para favorecer su formación desde unos contenidos de enseñanza. De la Herrán (2008) considera que la metodología pone de manifiesto las intenciones educativas del docente y sus premisas didácticas: concepción de educación, de enseñanza, de su didáctica específica, la idea que tiene del alumno, sus conocimientos aplicados a los elementos curriculares básicos (intenciones docentes, competencias, contenidos, criterios de evaluación) sus valores educativos, su capacidad para gestionar la motivación didáctica del alumnado, etc. El método didáctico es un camino por el que circula el alumno para alcanzar el conocimiento y el dominio de los contenidos realizando actividades de aprendizaje. Supone una acción dirigida y abierta, destinada a plantear una situación de aprendizaje, donde la relación interpersonal y el conocimiento comprensivo de la situación conduzcan a la creación de un proceso de aprendizaje; donde los sujetos formados lleguen a saber, hacer y actuar conforme la situación lo vaya exigiendo. El mejor método depende de su adecuación a la situación de enseñanza-aprendizaje: debe adaptarse al tipo y características de los alumnos, del profesor, de los recursos disponibles y el de la materia o habilidad que se debe enseñar.

Tabla 4: Valoración de la dimensión "Metodología docente"

\begin{tabular}{|l|c|c|c|}
\hline \multicolumn{1}{|c|}{ METODOLOGíA DOCENTE } & Media & $\mathbf{S}_{\mathbf{x}}$ & $\mathbf{N}$ \\
\hline $\begin{array}{l}\text { Organización y presentación de los materiales adecuada } \\
\text { (explicaciones, materiales escritos, actividades, etc.) }\end{array}$ & 3,60 &, 982 & 1309 \\
\hline $\begin{array}{l}\text { Coherencia entre los objetivos y contenidos de la asignatura y las } \\
\text { actividades realizadas }\end{array}$ & 3,63 &, 958 & 1304 \\
\hline Aporte de bibliografía y/o fuentes de información relevantes & 3,52 & 1,039 & 1302 \\
\hline Relación entre los distintos bloques temáticos de la materia & 3,69 &, 896 & 1277 \\
\hline $\begin{array}{l}\text { Coordinación entre el profesorado para evitar solapamientos y } \\
\text { Relacionar los contenidos de las distintas asignaturas }\end{array}$ & 3,11 & 1,281 & 1304 \\
\hline Seguimiento del proceso de aprendizaje del alumnado & 3,10 & 1,088 & 1302 \\
\hline Fomento de la participación en clase y motivación del alumnado & 3,25 & 1,110 & 1287 \\
\hline $\begin{array}{l}\text { Desarrollo de la capacidad de síntesis y de razonamiento del } \\
\text { alumnado }\end{array}$ & 3,35 & 1,305 & 1306 \\
\hline $\begin{array}{l}\text { Uso de metodologías para implicar activamente a los estudiantes } \\
\text { en el aprendizaje }\end{array}$ & 3,28 & 1,063 & 1308 \\
\hline $\begin{array}{l}\text { Organización en la exposición, destacando los contenidos más } \\
\text { importantes }\end{array}$ & 3,51 &, 994 & 1308 \\
\hline $\begin{array}{l}\text { Exposición de ejemplos en los que se ponen en práctica las } \\
\text { competencias a desarrollar por la asignatura }\end{array}$ & 3,55 & 1,001 & 1304 \\
\hline Promoción del desarrollo en el alumno de una actitud reflexiva & 3,32 & 1,016 & 1293 \\
\hline $\begin{array}{l}\text { Propuesta de actividades para favorecer el aprendizaje autónomo } \\
\text { (búsqueda de información complementaria, trabajos, } \\
\text { investigaciones, etc.) }\end{array}$ & 3,37 & 1,061 & 1307 \\
\hline Uso de un lenguaje claro, preciso y riguroso & 3,73 & 1,005 & 1306 \\
\hline cuestiones relacionadas con el desarrollo de la docencia & 3,14 & 1,108 & 1302 \\
\hline
\end{tabular}


La información aportada por la tabla 4 evidencia que todos los elementos que configuran esta dimensión han sido valorados de forma positiva por el alumnado encuestado. Sin embargo, es interesante establecer algún comentario más específico. Es por ello que los mejor valorados y, por lo tanto, incidirán en una mejora labor profesional del profesorado han sido los relacionados con aspectos didácticos el "uso de un lenguaje claro, preciso y riguroso", la "relación entre los distintos bloques temáticos de la materia", "la coherencia entre los objetivos y contenidos de la asignatura y las actividades realizadas" y la "organización y presentación de los materiales adecuada (explicaciones, materiales escritos, actividades, etc.)". En el polo opuesto, aunque no por ello valorados negativamente, destacan elementos más personales como el "seguimiento del proceso de aprendizaje del alumnado", "la coordinación entre el profesorado para evitar solapamientos y relacionar los contenidos de las distintas asignaturas" y "reparar en la opinión de los estudiantes en cuestiones relacionadas con el desarrollo de la docencia", quizá debido a ser las labores más desconocidas o menos realizadas por los y las docentes.

Para establecer alguna diferenciación en estas valoraciones atendiendo a la Universidad y a la macroárea de la que procede este alumnado y tras realizar un análisis de varianza (n.s.=0.05), se han obtenidos los siguientes resultados:

- El alumnado de la Universidad de Huelva considera relevante para una adecuada metodología docente todos los elementos indicados, a excepción de la "coherencia entre los objetivos y contenidos de la asignatura y las actividades realizadas", que es valorada del mismo modo por todas las universidades.

- El alumnado de la Universidad Jaume I manifiesta una gran importancia en todos los indicadores que conforman la dimensión, salvo en aspectos como la "coherencia entre los objetivos y contenidos de la asignatura y las actividades realizadas" (valorada del mismo modo por todas las universidades) y el "aporte de bibliografía y/o fuentes de información relevantes" y la "relación entre los distintos bloques temáticos de la materia" (valoradas en mayor medida por Huelva y Salamanca".

- El alumnado de la Universidad de Salamanca valora como positivos los siguientes elementos: "organización y presentación de los materiales adecuada (explicaciones, materiales escritos, actividades, etc.)", "aporte de bibliografía y/o fuentes de información relevantes", "relación entre los distintos bloques temáticos de la materia", "organización en la exposición, destacando los contenidos más importantes" y "uso de un lenguaje claro, preciso y riguroso".

- El alumnado de Humanidades manifiesta que la metodología docente ha de estar caracterizada, entre otros elementos, por: "aporte de bibliografía y/o fuentes de información relevantes", "organización en la exposición, destacando los contenidos más importantes", "propuesta de actividades para favorecer el aprendizaje autónomo (búsqueda de información complementaria, trabajos, investigaciones, etc.)" y "uso de un lenguaje claro, preciso y riguroso".

- El alumnado de titulaciones Jurídico-Sociales considera que la metodología docente ha de favorecer la "coordinación entre el profesorado para evitar solapamientos y relacionar los contenidos de las distintas asignaturas", el "seguimiento del proceso de aprendizaje del alumnado", el "fomento de la participación en clase y motivación del alumnado", el "uso de metodologías para implicar activamente a los estudiantes en el aprendizaje", la "organización en la exposición, destacando los contenidos más importantes", la "propuesta de actividades para favorecer el aprendizaje autónomo (búsqueda de información complementaria, trabajos, investigaciones, etc.)" y "reparar 
en la opinión de los estudiantes en cuestiones relacionadas con el desarrollo de la docencia".

- El alumnado de carreras Técnicas destaca, entre otros elementos, la "coordinación entre el profesorado para evitar solapamientos y relacionar los contenidos de las distintas asignaturas", la "organización en la exposición, destacando los contenidos más importantes" y "reparar en la opinión de los estudiantes en cuestiones relacionadas con el desarrollo de la docencia”.

- El alumnado de Ciencias de la Salud destaca en el "fomento de la participación en clase y motivación del alumnado" y en "reparar en la opinión de los estudiantes en cuestiones relacionadas con el desarrollo de la docencia".

\section{Actividades prácticas}

Se entiende esta dimensión como el diseño y desarrollo de prácticas vinculadas a los contenidos teóricos de las materias de la titulación para el desarrollo de las competencias profesionales.

Una valoración de los elementos que la configuran esta dimensión evidencia que todos son relevantes, en el sentido de que se aducen al contexto teórico de la materia, adaptadas al perfil profesional y en un número adecuado al tiempo disponible (ver tabla 5).

Tabla 5: Valoración de la dimensión "Actividades prácticas"

\begin{tabular}{|l|c|c|c|}
\hline \multicolumn{1}{|c|}{ ACTIVIDADES PRÁCTICAS } & Media & $\mathbf{S}_{\mathbf{x}}$ & $\mathbf{N}$ \\
\hline Adecuación de las prácticas al contexto teórico de la asignatura & 3,51 & 1,053 & 1304 \\
\hline Adecuación del número de prácticas al desarrollo de la asignatura & 3,30 & 1,170 & 1278 \\
\hline Utilidad de las prácticas para el desarrollo de competencias profesionales & 3,46 & 1,178 & 1301 \\
\hline
\end{tabular}

Al establecer alguna diferencia en función de la Universidad (Análisis de Varianza, n.s.=0.05), son las instituciones de Huelva y Jaume I de Castellón quienes más valoran este conjunto de indicadores.

Por otro lado y atendiendo a la macroárea, los resultados del Análisis de Varianza (n.s.=0.05) únicamente muestran diferencias en la valoración dada a la "utilidad de las prácticas para el desarrollo de competencias profesionales", siendo más elevada para los grupos de Ciencias de la Salud y Jurídico-Sociales.

\section{Recursos didácticos}

Se concibe como recurso didáctico cualquier recurso que el profesorado prevea emplear en el diseño o desarrollo del currículum (por su parte o por la de los alumnos) para aproximar o facilitar los contenidos, mediar en las experiencias de aprendizaje, provocar encuentros o situaciones, desarrollar habilidades cognitivas, apoyar sus estrategias metodológicas o facilitar o enriquecer la evaluación (Blázquez y Lucero, 2002).

Son valorados como positivos el empleo de todos aquellos recursos que tanto tradicionales como tecnológicos, faciliten el aprendizaje. Asimismo, se considera relevante disponer de un manual didáctico que acompañe en el desarrollo de las asignaturas (ver tabla 6). 
Tabla 6: Valoración de la dimensión "Recursos didácticos

\begin{tabular}{|l|c|c|c|}
\hline \multicolumn{1}{|c|}{ RECURSOS DIDÁCTICOS } & Media & $\mathbf{S}_{\mathbf{x}}$ & $\mathbf{N}$ \\
\hline $\begin{array}{l}\text { Uso de recursos adecuados (pizarra, transparencias, medios } \\
\text { audiosvisuales, material de apoyo en red virtual...) que facilitan el } \\
\text { aprendizaje }\end{array}$ & 3,80 & 1,006 & 1302 \\
\hline $\begin{array}{l}\text { Elaboración de manuales didácticos de apoyo al desarrollo de la } \\
\text { asignatura }\end{array}$ & 3,30 & 1,056 & 1304 \\
\hline Diseño de cuadernos de prácticas para su implementación en el aula & 3,10 & 1,173 & 1298 \\
\hline
\end{tabular}

Al igual que en los casos anteriores, hemos realizado un análisis de varianza (n.s. 00,05), obteniendo que son los estudiantes de la Universidad Jaume I de Castellón quienes realizan una valoración más elevada de todos los elementos que componen esta dimensión. Asimismo, el alumnado de Huelva también presta especial atención a los manuales didácticos y a los cuadernos de prácticas. También se advierte que solamente aparecen valoraciones más altas por parte del alumnado de carreras Jurídico-Sociales, Técnicas y Experimentales, al considerar como recurso prioritario el "Diseño de cuadernos de prácticas para su implementación en el aula".

Sistemas de evaluación

Se puede definir esta dimensión como el conjunto de procesos sistemáticos de recogida, análisis e interpretación de información relevante para medir o describir cualquier faceta de la realidad educativa y sobre esta descripción formular un juicio de valor con un criterio o patrón, todo ello como base para tomar decisiones (De la Orden, 1997). La evaluación, en su doble dimensión de apreciación del aprendizaje del alumno y de certificación de las habilidades alcanzadas, constituye otro eslabón esencial de la acción docente universitaria. La forma en que el profesorado universitario vive esta parte de su trabajo varía mucho de unos a otros y suele ser un excelente indicador de cómo perciben su papel de docentes, tal y como indica Zabalza (2009).

Tabla 7: Valoración de la dimensión "Recursos didácticos"

\begin{tabular}{|l|c|c|c|}
\hline \multicolumn{1}{|c|}{ SISTEMAS DE EVALUACIÓN } & Media & $\mathbf{S}_{\mathbf{x}}$ & $\mathbf{N}$ \\
\hline Información del sistema de evaluación al comienzo del curso & 3,81 & 1,058 & 1279 \\
\hline $\begin{array}{l}\text { Información del sistema de evaluación durante el transcurso } \\
\text { de la docencia }\end{array}$ & 3,58 & 1,066 & 1301 \\
\hline Información del sistema de evaluación al finalizar el curso & 3,50 & 1,158 & 1291 \\
\hline $\begin{array}{l}\text { Evaluación coherente con los objetivos, contenidos, } \\
\text { metodología y actividades del curso }\end{array}$ & 3,48 & 1,049 & 1297 \\
\hline $\begin{array}{l}\text { Variedad de procedimientos para evaluar el aprendizaje de } \\
\text { los alumnos/as }\end{array}$ & 3,26 & 1,099 & 1303 \\
\hline
\end{tabular}

Buscando diferencias atendiendo a la Universidad a la que pertenece este alumnado, se evidencian las siguientes:

- El alumnado de la Universidad de Huelva de forma más positiva que el del resto de universidades aprecia la existencia de una "variedad de procedimientos para evaluar el aprendizaje del alumnado".

- El alumnado de la Universidad Jaume I valora como relevantes todos los elementos que componen esta dimensión. Sin embargo, otorga menos relevancia a la dotación de 
"información del sistema de evaluación al finalizar el curso", siendo el alumnado de Salamanca quien revela este aspecto como más importante.

- Igualmente, los y las estudiantes de la Universidad de Salamanca consideran importantes elementos como "información del sistema de evaluación al comienzo del curso" e "información del sistema de evaluación durante el transcurso de la docencia".

La réplica del estudio anterior tomando en consideración la macroárea a la que pertenecen la titulación de los y las estudiantes, muestra que todas las actitudes son igualmente valoradas, a excepción del alumnado de estudios técnicos y experimentales, quien valora de un modo más alto que se aporte "información del sistema de evaluación al comienzo del curso".

\section{Actitud del profesorado}

Se considera que la actitud del profesorado aúna las creencias, pensamientos e ideas que influyen en la labor (actuaciones) docente y guía y orienta su quehacer, su labor.

Según la percepción del alumnado (ver tabla 8), los principales elementos actitudinales docentes pueden agruparse en profesionales (interés por la asignatura, promoción de la comunicación, clima de trabajo y participación y atención a las dificultades de aprendizaje) y personales (respeto y trato interpersonal).

Tabla 8: Valoración de la dimensión "Actitud del profesorado"

\begin{tabular}{|l|c|c|c|}
\hline \multicolumn{1}{|c|}{ ACTITUD DEL PROFESORADO } & Media & $\mathbf{S}_{\mathbf{x}}$ & $\mathbf{N}$ \\
\hline Actitud receptiva en su relación con el alumnado & 3,51 & 1,059 & 1296 \\
\hline Respeto hacia el alumnado & 3,98 & 1,022 & 1296 \\
\hline Interés por la asignatura por parte del docente & 3,82 & 1,009 & 1293 \\
\hline Atención ante las dificultades de aprendizaje de sus estudiantes & 3,48 & 1,133 & 1287 \\
\hline $\begin{array}{l}\text { Estímulo al alumnado para que se interese por su proceso de } \\
\text { aprendizaje }\end{array}$ & 3,39 & 1,102 & 1301 \\
\hline Creación de un clima de trabajo y participación & 3,50 & 1,081 & 1302 \\
\hline Promoción de una comunicación fluida y espontánea & 3,53 & 1,093 & 1302 \\
\hline
\end{tabular}

De nuevo y estableciendo diferencias en función de la universidad de procedencia del alumnado y de la macroárea a la que pertenecen, observamos que existen evidencias para establecer diferentes apreciaciones:

- El alumnado de la Universidad de Huelva valora muy positivamente todos los elementos de esta dimensión, a excepción del "respeto hacia el alumnado" que, aunque importante, es considerado de mayor relevancia por las instituciones de Salamanca y Jaume I.

- El alumnado de la Universidad de Salamanca valora como actitudes más importantes del profesorado universitario la "actitud receptiva en su relación con el alumnado" y el "respeto hacia el alumnado".

- El alumnado de la Universidad Jaume I de Castellón revela que todos los elementos actitudinales son positivos y necesarios para definir el perfil del profesorado universitario.

- El alumnado de titulaciones pertenecientes a las ramas de Experimentales y Humanidades valora como actitud prioritaria del profesorado el "respeto hacia el alumnado". 
- El alumnado de titulaciones pertenecientes a las ramas de Ciencias Jurídico-Sociales y Técnicas valora como actitud prioritaria del profesorado "creación de un clima de trabajo y participación".

\section{Conclusiones}

Tras la labor realizada a lo largo de las diferentes fases en las que se ha planificado este trabajo, cabe destacar que, habiendo atendido a la triple dimensión de las competencias del profesorado universitario: docente, investigadora y de gestión, los objetivos inicialmente marcados se han cubierto satisfactoriamente.

El protocolo de indicadores derivado de todo el trabajo hasta aquí descrito está configurado por un total de seis dimensiones descritas a lo largo de este documento, que engloban a los cuarenta indicadores que, de forma clara y precisa, representan al modelo docente que se pretende medir en las distintas facetas dentro de su actividad docente.

El perfil docente derivado del mismo responde a un profesional que desarrolla su actividad sobre la base de una labor planificada de forma sistemática y con suficiente antelación, donde la información a transmitir está ligada directamente con las competencias profesionales del trabajo para el que capacita la titulación en la que se encuentra y que emplea recursos, metodologías y estrategias didácticas en un contexto específico de enseñanza-aprendizaje donde la planificación docente, la gestión del tiempo y del espacio, los sistemas de evaluación y la interacción con el alumnado juegan un papel importante.

Se configura como una propuesta en consonancia con las reflexiones desarrolladas por Fuentes y Herrero (1999), que argumentaron la necesidad de propuestas de evaluación docente que se basen un profesor o profesora integral, un sistema que apunte a la labor instructiva, investigadora, cooperativa y divulgativa; en definitiva, un modelo que considere de forma completa su desarrollo profesional.

\section{Bibliografía}

Blázquez, F. y Lucero, M. (2002). Los medios y recursos en el proceso didáctico. En A. Medina. y F. Salvador (Coords.). Didáctica general. Madrid: Prentice Hall.

De la Herrán, A. (2008). Metodología didáctica en educación secundaria: una perspectiva desde la Didáctica General. En A. De la Herrán y J. Paredes (Coord.). Didáctica General. Madrid: McGraw-Hill.

De la Orden, A. (1997). Desarrollo y validación de un modelo de calidad universitaria como base para su evaluación. Revista Electrónica de Investigación y Evaluación Educativa, 3. Consultado el 25 de julio de 2011 en http://www.uv.es/RELIEVE/v3n1/RELIEVEv3n1_2.htm

Declaración de Bolonia (1999). The European Higher Education Area. Bologna Declaration. Joint declaration of the European Ministers of Education. Bolonia, 19 de junio de 1999. Consultado el 4 de febrero de 2009 en http://www.univ.mecd.es/univ/html/informes/bolonia/Declaracion_Bolonia.pdf.

Fuentes, M.E. y Herrero, J.R. (1999). Evaluación docente: hacia una fundamentación de la autoevaluación. Revista Electrónica Interuniversitaria de Formación del profesorado, 2 (1), 353-368. Consultado el 13 de julio de 2011 en http://www.uva.es/aufop/publica/revelfop/99-v2n1.htm 
Fueyo, A. (2004). Evaluación de titulaciones, centros y profesorado en el proceso de convergencia Europea: ¿de qué calidad y de qué evaluación hablamos?. Revista Interuniversitaria de Formación del Profesorado, 18 (3), 207-219.

Jornet, J. et al. (1988). Proyecto de implantación de un sistema de evaluación del profesorado en la Universidad de Valencia. En Universidad de Valencia. Consideraciones metodológicas sobre la evaluación y mejora de la docencia universitaria. Informes de Investigación Evaluativa, 1. Valencia: Universidad de Valencia.

Perrenoud, P. (2004). Diez nuevas competencias para enseñar: invitación al viaje. Barcelona: Graó.

Rué, J. (2004). Conceptuar el aprendizaje y la docencia en la universidad mediante los ETCS. Revista Interuniversitaria de Formación del Profesorado, 18 (3). 179-195.

Salinas, D. y Cotino C. (2005). Elaboración de la Guía Docente para la Convergencia Europea. Principios para su diseño. Valencia: Universidad de Valencia.

Tejedor, F.J. y Jornet, J.M. (2008). La evaluación del profesorado universitario en España. Revista Electrónica de Investigación Educativa. Consultado el 15 de marzo de 2011 en http://redalyc.uaemex.mx/src/inicio/ArtPdfRed.jsp?iCve=15511127005.

Tiana Ferrer, A. (1998). Indicadores educativos. Qué son y qué pretenden. Cuadernos de Pedagogía, 256, 51-53.

Zabalza, M.A. (2007). La enseñanza universitaria. El escenario y sus protagonistas. Madrid: Narcea.

Zabalza, M.A. (2009). Competencias docentes del profesorado universitario. Calidad y desarrollo profesional. Madrid: Narcea. 


\section{Autores}

Ana Belén López Cámara

Becaria de colaboración para la formación práctica en la implantación de títulos de Grado y en la innovación docente derivada de la aplicación del sistema de créditos europeos durante el Curso 2011-2012 en la Facultad de Ciencias de la Educación de la Universidad de Córdoba. Es Licenciada en Psicopedagogía por la Facultad de Ciencias de la Educación, Universidad de Córdoba. Centra su investigación en la elaboración de taxonomías competenciales del profesorado universitario y participa activamente en el Grupo de Investigación SEJ 049 (Evaluación Educativa e Innovación) en proyectos de investigación y trabajo relacionados con la atención a la diversidad, la evaluación de competencias y la creación de comunidades de aprendizaie en ambientes desfavorecidos

Ignacio González López

Profesor Titular de Universidad en el Área de Métodos de Investigación y Diagnóstico en Educación. Ocupa actualmente el cargo de Vicedecano de Planes de Estudios de la Facultad de Ciencias de la Educación, así como la Coordinación del Máster de Educación Inclusiva de eta universidad. Es coordinador del Grupo de Investigación SEJ049 (Evaluación Educativa e Innovación) que centra su trabajo en proyectos de investigación y trabajo relacionados con la atención a la diversidad, la evaluación de competencias docentes del profesorado universitario y la creación de comunidades de aprendizaje en ambientes desfavorecidos. Su investigación está centrada en aspectos tales como la medición y evaluación educativa, la formación en competencias y la mejora de la calidad educativa

Carlota de León Huertas

Doctora por la Universidad de Córdoba, es profesora colaboradora del Área de Didáctica y Organización Educativa en Educación en la Universidad de Córdoba. Actualmente es coordinadora de la Licenciatura de Pedagogía. Ha colaborado como investigadora en diferentes proyectos de investigación, innovación y formación del profesorado destacando: La transición escuela obligatoria- trabajo. Los departamentos de orientación de los IES y la inserción laboral de los alumnos que abandonan la escuela tras la ESO; Estudiantes en riesgo de exclusión educativa en la ESO: Situación, Programas y buenas prácticas en la Comunidad Autónoma de Andalucía. Participa activamente en el Grupo de Investigación SEJ 049 (Evaluación Educativa e Innovación) en proyectos de investigación y trabajo relacionados con la atención a la diversidad, la evaluación de competencias docentes del profesorado universitario y la creación de comunidades de aprendizaje en ambientes desfavorecidos 